\title{
Effect of evaluation frequency separation on magnetotelluric resolution
}

\author{
Ujjal K. Borah $^{1,{ }^{\star}}$, Prasanta K. Patro ${ }^{1}$
}

${ }^{1}$ Magnetotellurics and Deep Resistivity Sounding Division CSIR - National Geophysical Research Institute, Hyderabad, India

\author{
Article history \\ Received May 18, 2016; accepted March 02, 2017. \\ Subject classification: \\ Magnetotelluric, Resolution, Electromagnetic, Diffusion, Skin depth.
}

\begin{abstract}
Aiming at the resolution enhancement of the subsurface objects, an evaluation (target) frequency separation criterion is proposed for magnetotelluric (MT) method. The proposed idea is based on the propagation geometry of diffused electromagnetic (EM) wave through the earth. Starting from a homogeneous medium and extended it to layered earth model, the present study proposed the frequency separation criterion in terms of apparent resistivity and the lowest frequency to be used. To apply the proposed idea in real situation the frequency separation criterion is reduced to a convenient form which is expressed in terms of the lowest frequency only. The effectiveness of the proposed condition is examined by applying it to synthetic data. The result shows that the new idea can improve the resolution of the subsurface objects.
\end{abstract}

\section{Introduction}

Magnetotelluric (MT), a passive electromagnetic (EM) method, was introduced by Louis Cagniard in early 50s [Cagniard 1953]. MT is a diffusive EM method which implies that it is volumetric measurement method [Simpson \& Bahr 2005]. So, this diffusive volumetric measurement at a particular frequency gives the information within a hemisphere whose radius is equal to the skin depth for that frequency. As a result, the resolution of MT measurement is frequency dependent. So, while investigating with MT method the resolvability of an target object at a certain depth depends on skin depth [Thiel et. al. 2010]; in turn on measured frequency and resistivity of the overlying material [Orange 1989] and is decreasing with increasing depth [Peacock et. al. 2012]. Basically, the resolution of an individual layer depends on resistivity contrast with the surrounding, thickness of the layer and depth from the surface [Orange 1989]. Many researchers [Bedrosian 2007, Jones 1987, Orange 1989, Thiel et. al. 2010] have addressed this resolu- tion issue qualitatively. So, in the present study a quantitative attempt has been made to find the minimum resolvable layer thickness at a certain depth and the maximum evaluation (target) frequency separation for resolution enhancement.

The study is based on the propagation geometry of the diffused EM wave through the earth. This concept gives the EM wave a hemispherical shape for a particular frequency with a fixed radius which is the skin depth of that frequency. The difference between the radiuses of two hemispheres i.e. skin depths of two evaluation (target) frequencies is taken for the calculation of minimum resolvable layer thickness at a particular depth. This difference expression is a function of frequency. So, we applied the concept of differentiation to calculate the maximum evaluation frequency separation for resolution enhancement of the layer. This approach is first applied to a homogeneous earth where the shape of the diffusive EM wave takes the perfect hemispherical shape and deduced the preliminary condition for maximum separation of evaluation frequency. After achieving this, we extended the homogeneous earth situation to a layered earth situation. In layered earth situation the diffused EM waves do not take perfect hemispherical shape as distortion causes at the boundaries. So, in this case we have derived maximum frequency separation condition taking the apparent resistivities related to the target frequencies into account. Finally the derived criterion is tested with synthetic data to verify its effectiveness. Also we have studied the enhancement of horizontal resolution on the basis of overlapping of diffused hemispheres from two nearby stations and derived the formulation for horizontal resolution in terms of station spacing (Appendix A). 


\section{Methodology:}

\subsection{Vertical resolution:}

When an EM wave propagates through a medium then its amplitude is decreasing exponentially and the depth at which the amplitude of this exponentially decaying EM wave reduced to $\left(\frac{1}{\mathrm{e}}\right)$ times $(\approx 37 \%)$ that of its surface value is the Skin depth. Considering the basic assumption of MT the skin depth for this study is taken as $\delta=\sqrt{\frac{2}{\omega \mu \sigma}} \approx 500 \sqrt{\frac{\rho}{f}}$ where $\omega$ is the angular frequency, $\mu$ is the magnetic permeability and $\sigma$ is the conductivity of the medium of propagation of the EM wave.

Now, consider two evaluation (target) frequencies $f_{m}$ and $f_{n}\left(f_{m}>f_{n}\right)$ for MT investigation in a homogeneous medium of resistivity $\rho$ (Figure 1a).

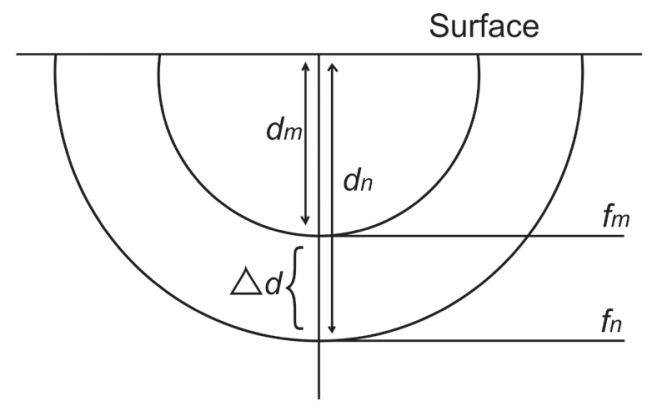

$1(a)$

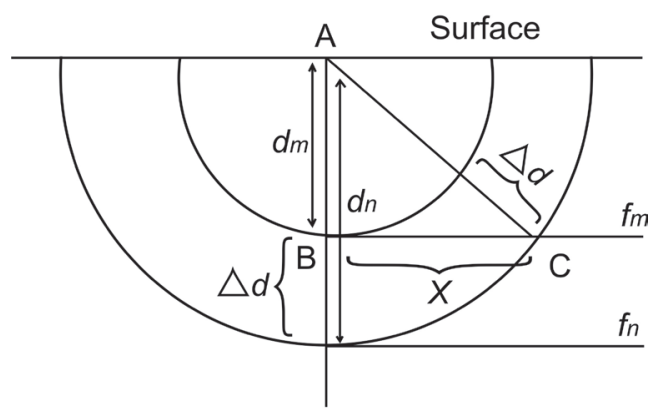

$1(b)$

Figure 1. Propagation geometry of diffusive EM wave in homogeneous medium. 1(a) Hemispherical shape of diffuse EM wave for two frequencies $f_{m}$ and $f_{n}\left(f_{m}>f_{n}\right) \cdot d_{m}$ and $d_{n}$ are the corresponding skin depths and $\Delta d=d_{m}-d_{n}$ is the difference of skin depths, provides the minimum resolvable thickness for that two frequencies. 1(b) Minimum horizontal resolvable length $x(=\mathrm{BC})$ with two frequencies $f_{m}$ and $f_{n}$ below a depth $d_{m}$ on one side of the station $A$.

Then their corresponding skin depths will be

$d_{m}=500 \sqrt{\frac{\rho}{f_{m}}}$ and $d_{n}=500 \sqrt{\frac{\rho}{f_{n}}}$ with $d_{n}>d_{m}$

So, for this homogeneous medium the separation between the skin depths of frequencies $f_{m}$ and $f_{n}$ below a depth $d_{m}$ (Figure 1a) will be

$$
\Delta d=500 \sqrt{\frac{\rho}{f_{n}}}-500 \sqrt{\frac{\rho}{f_{m}}}
$$

For a constant resistivity value this depth separation is as a function of frequency

$$
\Delta d=g(f)=500 \sqrt{\frac{\rho}{f_{n}}}-500 \sqrt{\frac{\rho}{f_{m}}}=500 \sqrt{\rho}\left[\frac{1}{\sqrt{f_{n}}}-\frac{1}{\sqrt{f_{m}}}\right]
$$

The function value always tends to increase when the frequencies are changed from high to low and vice-versa. So, this function does not have a fixed minimum or maximum; as the value proceeds either towards lower or higher end when the frequency separation is changed. But for a constant depth separation i.e. for constant $\Delta d$, we can find the optimal frequency separation condition by differentiating the function w.r.t frequency ( $f$ ). So, for a fixed $\Delta d$ we have the condition for optimal frequency separation as

$$
\begin{gathered}
\frac{d}{d f}\left[500 \sqrt{\rho}\left\{\frac{1}{\sqrt{f_{n}}}-\frac{1}{\sqrt{f_{m}}}\right\}\right]=0 \\
500 \sqrt{\rho}\left[\frac{d}{d f}\left(\frac{1}{\sqrt{f_{n}}}\right)-\frac{d}{d f}\left(\frac{1}{\sqrt{f_{m}}}\right)\right]=0
\end{gathered}
$$

As in MT, evaluation frequencies are represented in logarithmic scale, so these two evaluation frequencies $\left(f_{m} \& f_{n}\right)$ in log scale can be related as

$$
\begin{aligned}
& f_{m}=a f_{n}^{k} \quad\left(\text { for frequencies }>1 \mathrm{~Hz} ; f_{m}>f_{n}\right) \\
& f_{m}=a f_{n}^{1 / k} \quad\left(\text { for frequencies }<1 \mathrm{~Hz} ; f_{m}>f_{n}\right)
\end{aligned}
$$

Where ' $a$ ' is constant and ' $k$ ' is power on lower frequency. Plotting of this relation will give a straight line whose intercept is $a$ and slope is $k$ or $(1 / k)$.

Case 1: If $f_{m}$ and $f_{n}>1 \mathrm{~Hz}$ i.e. $f_{m}=a f_{n}^{k}$

Putting the value of $f_{m}$ from Equation (6) into Equation (5) we have

$$
\begin{gathered}
500 \sqrt{\rho}\left[\frac{d}{d f}\left(\frac{1}{\sqrt{f_{n}}}\right)-\frac{d}{d f}\left(\frac{1}{\sqrt{a f_{n}^{k}}}\right)\right]=0 \\
\frac{d}{d f}\left(f_{n}^{-\frac{1}{2}}\right)-\frac{1}{\sqrt{a}} \frac{d}{d f}\left(f_{n}^{\frac{-k}{2}}\right)=0
\end{gathered}
$$




$$
\begin{gathered}
\left(-\frac{1}{2}\right) f_{n}^{\left(\frac{-1}{2}\right)-1}=\frac{1}{\sqrt{a}\left(-\frac{k}{2}\right) f_{n}^{\left(-\frac{k}{2}\right)-1}} \\
\frac{1}{\sqrt{f_{n}}}=\frac{k}{\sqrt{a f_{n}^{k}}}
\end{gathered}
$$

Replacing $a f_{n}^{k}$ by $f_{m}$ (from Equation 6) and simplifying

$$
f_{m}=k^{2} f_{n}
$$

Subtracting $f_{n}$ from both sides

$$
\begin{gathered}
f_{m}-f_{n}=k^{2} f_{n}-f_{n} \\
\Delta f=\left(k^{2}-1\right) f_{n}
\end{gathered}
$$

where $\Delta f=f_{m}-f_{n}$ is the frequency separation.

Now, we have to find the value of $k$. For that we related the $n^{\text {th }}$ evaluation frequency of a decade with the sampling frequency $\left(f_{s}\right)$ as [Simpson \& Bahr 2005]-

$$
f_{n}=\frac{\left(f_{s} / N\right)}{\sqrt{2^{n-1}}} ; \quad \mathrm{N}=2,3,4 \ldots
$$

But according to the Nyquist rule we have minimum $N$ value as 2 . So, under this minimum condition

$$
f_{n}=\frac{f_{s}}{2 \sqrt{2^{n-1}}}
$$

Again for the $\mathrm{m}^{\text {th }}$ evaluation frequency

$$
f_{m}=\frac{f_{s}}{2 \sqrt{2^{m-1}}}
$$

As we are looking for the evaluation frequency separation, so, $f_{n}$ and $f_{m}$ should be consecutive evaluation frequencies from a decade. So, for a particular decade

$$
f_{n}=\frac{f_{s}}{2 \sqrt{2^{n-1}}} \text { and } f_{m}=f_{n-1}=\frac{f_{s}}{2 \sqrt{2^{n-2}}}
$$

Putting the value of $f_{m}$ and $f_{n}$ in Equation (12) we have

$$
\begin{aligned}
\frac{f_{s}}{2 \sqrt{2^{n-2}}} & =k^{2} \frac{f_{s}}{2 \sqrt{2^{n-1}}} \\
k^{2} & =\sqrt{2}
\end{aligned}
$$

In case of homogeneous earth we have ideal hemispherical condition. But as soon as we are dealing with layered earth situation the hemispherical condition is violated (Figure 2) at the boundaries between different layers. The diffusive wave will take a position upper or lower than the original ideal hemispherical condition (Figure 2) depending on the resistivity contrast of the corresponding layers. So, during the calculation of vertical resolution at a certain depth we have to take care of this distortion. In Figure 3, we have the

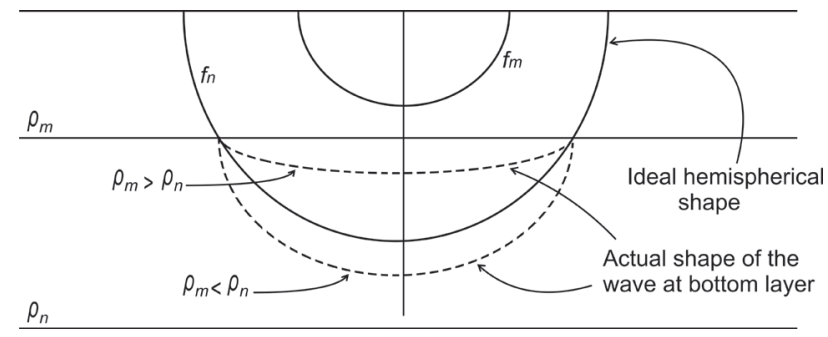

Figure 2. Effect of 1D earth on hemispherical shape of diffusive EM wave. Distortion of hemispherical shape of EM wave occurs due to the resistivity contrast between top and bottom layer. Hemisphere corresponds to frequency $f_{m}$ confined within the top layer has no distortion while the hemisphere corresponds to frequency $f_{n}$ enters into the bottom layer experiences distortion. The solid black colored line shows the ideal hemispherical shape and the dotted black colored lines show the distorted shape of the hemisphere. If $\rho_{m}>\rho_{n}$ then the hemisphere will take a position upward and if $\rho_{m}<\rho_{n}$ then the hemisphere will take position downward w.r.t the ideal shape at the bottom layer.

distorted situation of the diffusive wave. Here, the solid black colored line represents the actual position of the wave at subsurface and the red colored line represents the assumed hemispherical shape of the wave for that skin depth. Vertical resolvable thickness is affected by this distortion as the geometry of the diffusive wave is deviated from proper hemispherical shape. While dealing with homogeneous earth we have used single resistivity value. But in case of layered earth we have to use apparent resistivity for each corresponding frequencies. So, for a 1D layered earth we have the condition for optimal frequency separation (from Equation 4) as

$$
\frac{d}{d f}\left[500\left\{\sqrt{\frac{\rho_{n}^{a}}{f_{n}}}-\sqrt{\frac{\rho_{m}^{a}}{f_{m}}}\right\}\right]=0
$$

Where $\rho_{n}^{a}$ and $\rho_{m}^{a}$ are the apparent resistivities correspond to the evaluation frequencies $f_{n}$ and $f_{m}$. The values of apparent resistivities $\left(\rho_{m}^{a} \& \rho_{n}^{a}\right)$ will also change with the change of frequency. So, to handle this situation let us consider a common resistivity $(\rho ')$ and two multipliers $b_{1}$ and $b_{2}$ such that

$$
\begin{aligned}
& \rho_{m}^{a}=b_{1} \rho^{\prime} \text { and } \rho_{n}^{a}=\frac{\rho^{\prime}}{b_{2}} \\
& b_{1} b_{2}=\left\{\begin{array}{lll}
\left(\frac{\rho_{m}^{a}}{\rho_{n}^{a}}\right) & \text { if } & \rho_{m}^{a} \geq \rho_{n}^{a} \\
\left(\frac{\rho_{n}^{a}}{\rho_{m}^{a}}\right) & \text { if } & \rho_{m}^{a}<\rho_{n}^{a}
\end{array}\right.
\end{aligned}
$$

Now simplifying Equation (21) with Equation (22) we have

$$
500 \frac{d}{d f}\left[\left\{\sqrt{\frac{\left(\frac{\rho^{\prime}}{b_{2}}\right)}{f_{n}}}-\sqrt{\frac{b_{1} \rho^{\prime}}{f_{m}}}\right\}\right]=0
$$




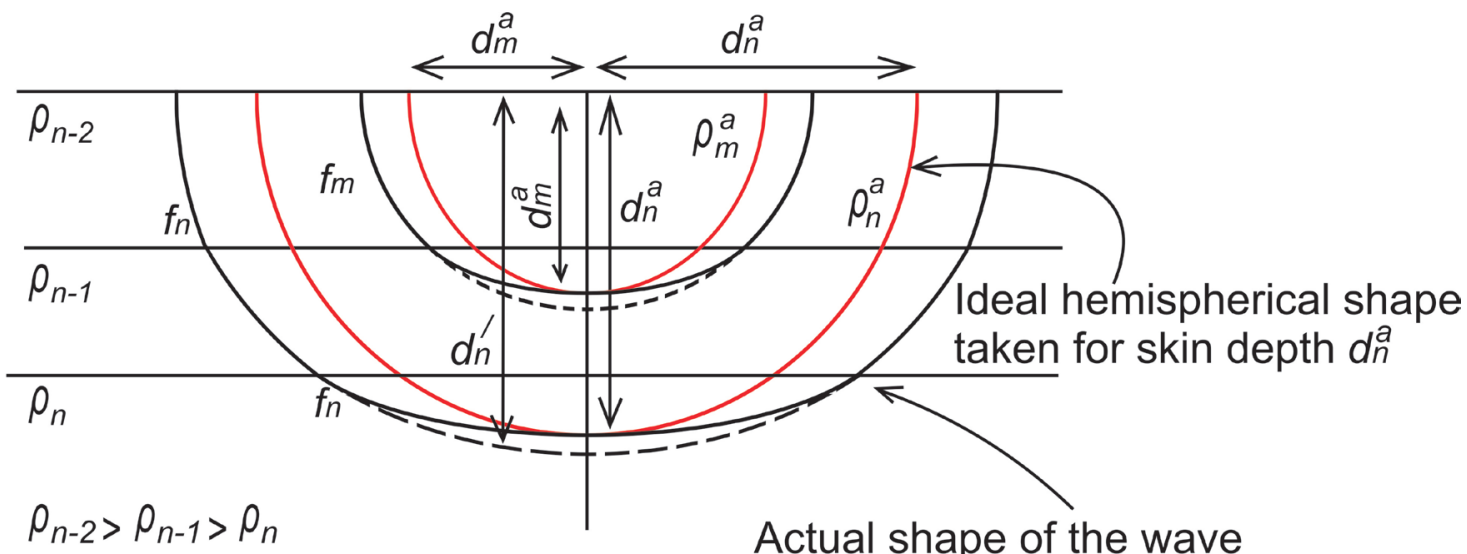

$3(\mathrm{a})$

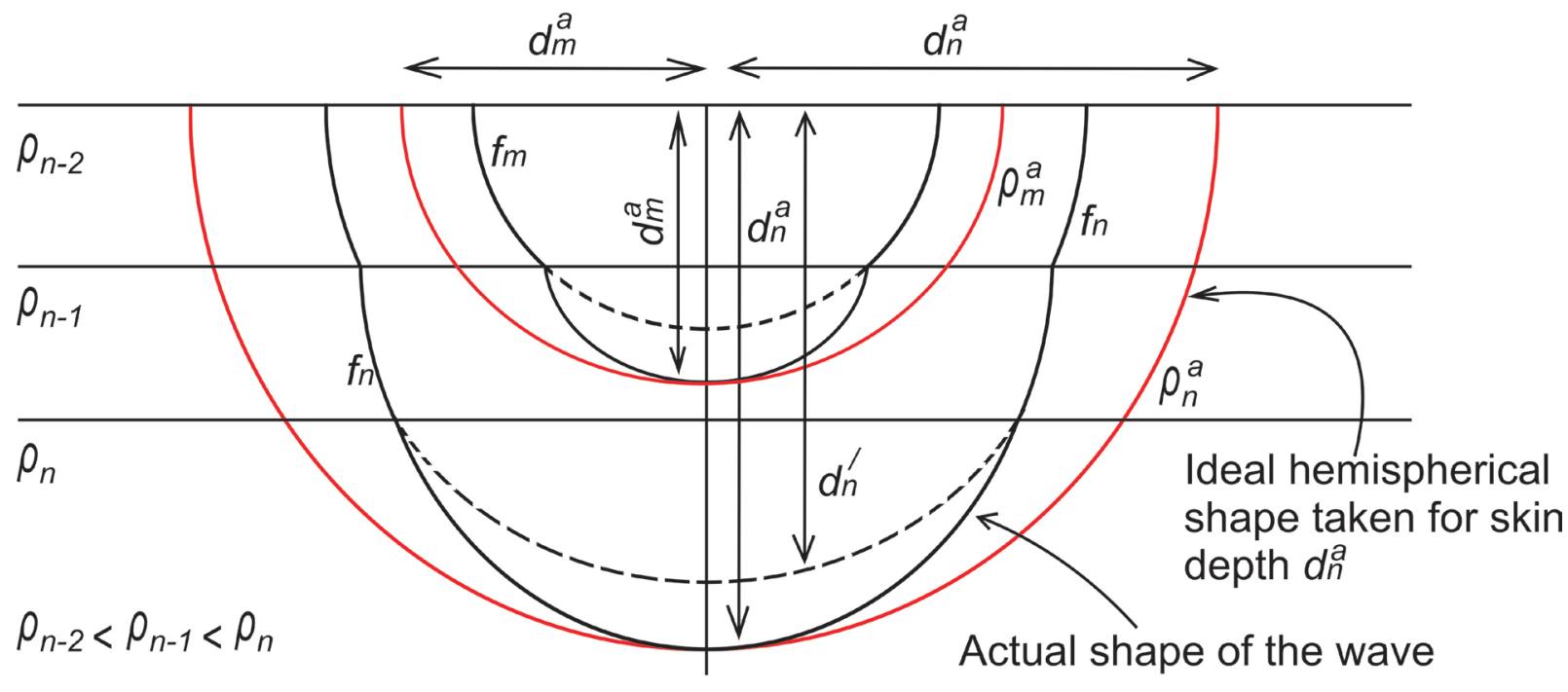

$3(b)$

Figure 3. Actual and assumed position of hemisphere corresponds to a skin depth of a particular frequency. $f_{n}, f_{m}$ are the evaluation frequencies and $d_{n}^{a}, d_{m}^{a}$ are the corresponding skin depths. 3 (a) Position of hemisphere in a three layered earth where the resistivity of the upper layer $\left(\rho_{n-1}\right)$ is greater than the resistivity of the lower layer $\left(\rho_{n}\right)$ and so on $\left(\rho_{n-2}>\rho_{n-1}>\rho_{n}\right)$. The black colored solid line for frequency $f_{n}$ and $f_{m}$ show the actual shape of the wave and the red colored solid line represent the assumed hemispherical shape of the wave for that skin depth $d_{n}^{a}$, and $d_{m}^{a} 3$ (b) Position of hemisphere in a three layered earth where the resistivity of the upper layer $\left(\rho_{n-1}\right)$ is less than the resistivity of the lower layer $\left(\rho_{n}\right)$ and so on $\left(\rho_{n-2}<\rho_{n-1}<\rho_{n}\right)$. The black colored solid line for frequency $f_{n}$ and $f_{m}$ represent the actual shape of the wave at a skin depth $d_{n}^{a}$ and $d_{m}^{a}$ and the red colored solid line shows the assumed hemispherical shape of the wave for that skin depths.

Putting the value of $f_{m}$ from Equation (6) in Equation (24) we have

$$
\begin{gathered}
{\left[\frac{d}{d f}\left(\sqrt{\frac{\left(\frac{\rho^{\prime}}{b_{2}}\right)}{f_{n}}}\right)-\frac{d}{d f}\left(\sqrt{\frac{b_{1} \rho^{\prime}}{a f_{n}^{k}}}\right)\right]=0} \\
{\left[\left(\sqrt{\frac{1}{b_{2}}}\right) \frac{d}{d f}\left(\frac{1}{\sqrt{f_{n}}}\right)-\left(\sqrt{\frac{b_{1}}{a}}\right) \frac{d}{d f}\left(\frac{1}{\sqrt{f_{n}^{k}}}\right)\right]=0} \\
\left(\sqrt{\frac{1}{b_{2}}}\right) \frac{d}{d f}\left(f_{n}^{-1 / 2}\right)-\left(\sqrt{\frac{b_{1}}{a}}\right) \frac{d}{d f}\left(f_{n}^{-k / 2}\right)=0
\end{gathered}
$$

$$
\begin{gathered}
\left(-\frac{1}{2}\right) f_{n}^{\left(-\frac{1}{2}\right)^{-1}}=\left(\sqrt{\frac{b_{1} b_{2}}{a}}\right)\left(-\frac{k}{2}\right) f_{n}^{\left(-\frac{k}{2}\right)-1} \\
\frac{1}{\sqrt{f_{n}}}=\frac{\left(\sqrt{b_{1} b_{2}}\right) k}{\sqrt{a f_{n}^{k}}}
\end{gathered}
$$
fying

Replacing $a f_{n}^{k}$ by $f_{m}$ (from Equation 6) and simpli-

$$
f_{m}=k^{2}\left(b_{1} b_{2}\right) f_{n}
$$

Now, if $\rho_{m}^{a} \geq \rho_{n}^{a}$

then

$$
f_{m}=k^{2}\left(\frac{\rho_{m}^{a}}{\rho_{n}^{a}}\right) f_{n}
$$


Subtracting $f_{n}$ from both sides

$$
\begin{gathered}
f_{m}-f_{n}=k^{2}\left(\frac{\rho_{m}^{a}}{\rho_{n}^{a}}\right) f_{n}-f_{n} \\
\Delta f=\left\{k^{2}\left(\frac{\rho_{m}^{a}}{\rho_{n}^{a}}\right)-1\right\} f_{n}
\end{gathered}
$$

Putting the value of ' $k$ ' (from Equation 20) we have

$$
\Delta f=\left\{\sqrt{2}\left(\frac{\rho_{m}^{a}}{\rho_{n}^{a}}\right)-1\right\} f_{n}
$$

Similarly, if $\quad \rho_{m}^{a}<\rho_{n}^{a}$

Then

$$
\Delta f=\left\{\sqrt{2}\left(\frac{\rho_{n}^{a}}{\rho_{m}^{a}}\right)-1\right\} f_{n}
$$

Where $\Delta f=f_{m}-f_{n}$ is the maximum frequency separation. So, to enhance the resolution the frequency separation should be less than the separation given by Equations (34), (35).

Hence, condition for evaluation (target) frequency separation is

$$
\left.\begin{array}{l}
\Delta f \leq\left\{\sqrt{2}\left(\frac{\rho_{m}^{a}}{\rho_{n}^{a}}\right)-1\right\} f_{n} ;\left(\text { for } \rho_{m}^{a}>\rho_{n}^{a}\right) \\
\Delta f \leq\left\{\sqrt{2}\left(\frac{\rho_{n}^{a}}{\rho_{m}^{a}}\right)-1\right\} f_{n} ;\left(\text { for } \rho_{m}^{a}<\rho_{n}^{a}\right)
\end{array}\right\}
$$

Case 2: If $f_{m}$ and $f_{n}<1 \mathrm{~Hz}$ i.e. $f_{m}=a f_{n}^{\frac{1}{k}}$

Proceeding similarly as described above we can have for this frequency range in homogeneous medium

$$
f_{m}=\left(\frac{1}{k^{2}}\right) f_{n}
$$

Which gives the value of ' $k$ ' for two consecutive frequencies as

$$
k^{2}=\frac{1}{\sqrt{2}}
$$

and the evaluation frequency separation as

$$
\left.\begin{array}{l}
\Delta f \leq\left\{\frac{1}{k^{2}}\left(\frac{\rho_{m}^{a}}{\rho_{n}^{a}}\right)-1\right\} f_{n}=\left\{\sqrt{2}\left(\frac{\rho_{m}^{a}}{\rho_{n}^{a}}\right)-1\right\} f_{n} ;\left(\text { for } \rho_{m}^{a}>\rho_{n}^{a}\right) \\
\Delta f \leq\left\{\frac{1}{k^{2}}\left(\frac{\rho_{n}^{a}}{\rho_{m}^{a}}\right)-1\right\} f_{n}=\left\{\sqrt{2}\left(\frac{\rho_{n}^{a}}{\rho_{m}^{a}}\right)-1\right\} f_{n} ;\left(\text { for } \rho_{m}^{a}<\rho_{n}^{a}\right)
\end{array}\right\}
$$

So, for all the MT frequency range we have the same evaluation frequency separation condition.

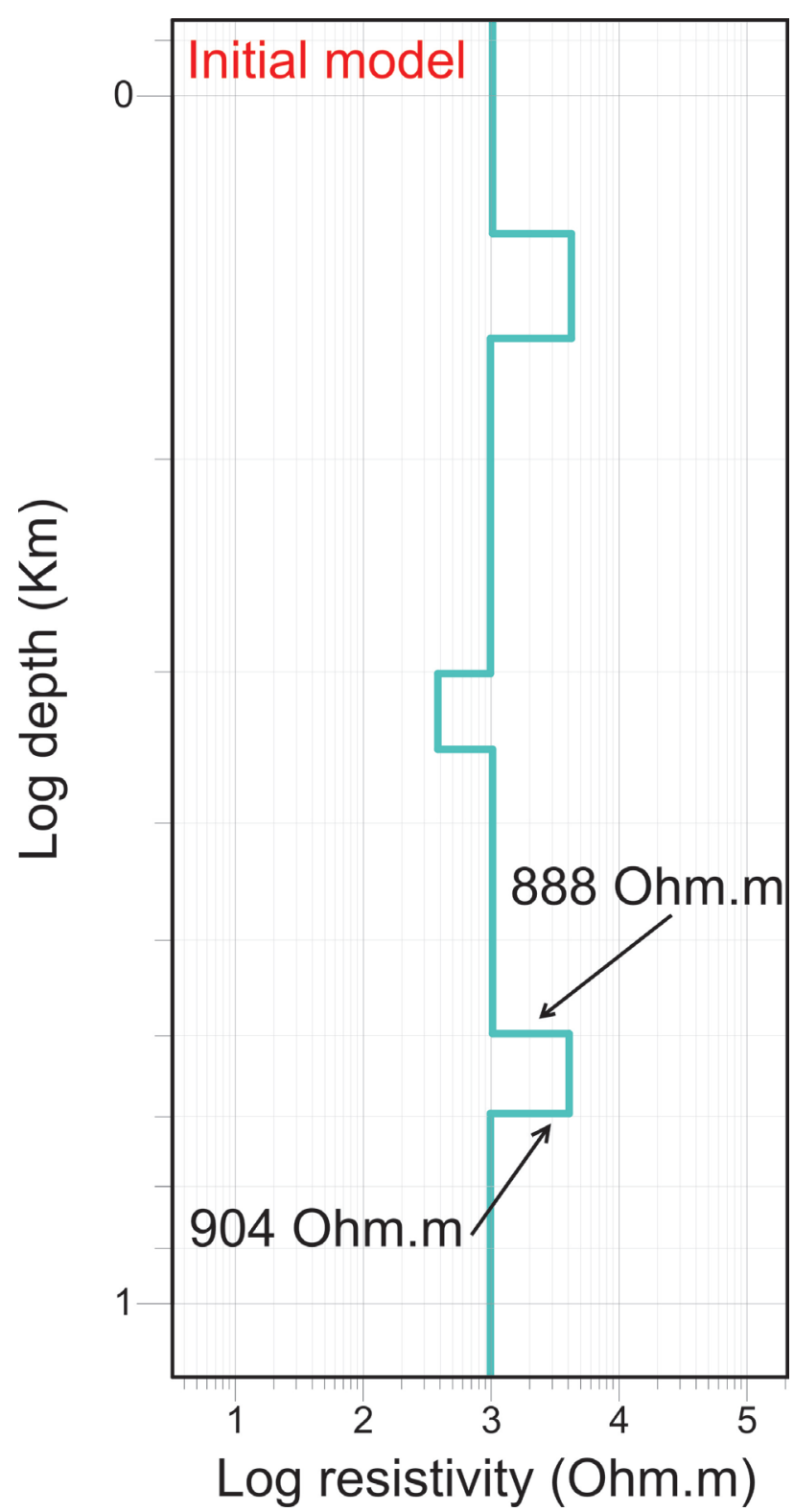

Figure 4. Initial synthetic 1D model to test the evaluation frequency separation condition. Two high resistive layers $(4000 \Omega \mathrm{m})$ and a low resistive layer $(400 \Omega \mathrm{m})$ are embedded within a common background of $1000 \Omega \mathrm{m}$. The apparent resistivities calculated [Spies 1989] upto the top and the bottom of the lowermost layer are obtained as $888 \Omega \mathrm{m}$ and $904 \Omega \mathrm{m}$ respectively.

\subsection{Synthetic test for evaluation frequency separation} condition

The proposed condition for separation of evaluation frequencies (Equations 36, 39) is studied with 1D synthetic data. Figure (4) shows an initial model taken for the resolution study. Here, two high resistive layers $(4000 \Omega \mathrm{m})$ and a comparatively low resistive layer (400 $\Omega \mathrm{m})$ are embedded within a common background $(1000 \Omega \mathrm{m})$. Synthetic data is generated from it and this data is tested for vertical resolution using two sets of evaluation frequencies (Table 1), one set is taken according to the proposed frequency separation criteria (Equations 36, 39) - set $\mathrm{A}$ and the other is not - set $\mathrm{B}$. 
First we calculated the apparent resistivity for the lower most layer using the concept of cumulative and average conductivity [Spies 1989]. After calculation, we got the apparent resistivity upto top of the bottom layer as 888 $\Omega \mathrm{m}$ and upto the bottom of the layer as $904 \Omega \mathrm{m}$ (Figure 4). Then we calculated the maximum frequency separation for these two apparent resistivities (from equation 35) and we found it as $0.44 f_{n}$. So, we took it as $0.3 f_{n}$ for frequency set A (to satisfy the equations 36,39 ) and $0.6 f_{n}$ for frequency set $\mathrm{B}$.

First, generated synthetic data is inverted using Occam inversion scheme [Constable et. al 1987]. From this multilayered model the approximate layers are outlined by fitting the model responses with the synthetic data. After that, to get a prominent fit of the model responses with the synthetic data Marquardt inversion [Marquard 1963] was run. The final inverted model obtained with frequency set $\mathrm{A}$ is 'model $\mathrm{A}$ ' (Figure $5 \mathrm{~b}$ ) and with frequency set B is 'model B' (Figure 5c). While doing a comparative study between the model A and model B with the initial model it is clearly seen that the layers are resolved more prominently in case of model A. Figure (7) provides a comparison between model A and model $B$ with the initial model.

\section{Discussion:}

From the above study it is established that the proposed criteria can enhance the resolution (both of resistivity and layer thickness) of subsurface structure upto certain extant. But question is how to apply the proposed criteria in real situation; as in real situation the resistivities of subsurface layers are unknown. To solve this problem we propose the following approach.

We have to deal with resistivities $\rho_{m}^{a}$ and $\rho_{n}^{a}$.

If $\rho_{m}^{a}>>\rho_{n}^{a}$ or $\rho_{m}^{a}<<\rho_{n}^{a}$ then

$$
\frac{\rho_{m}^{a}}{\rho_{n}^{a}} \rightarrow \infty \quad \text { or } \quad \frac{\rho_{n}^{a}}{\rho_{m}^{a}} \rightarrow \infty
$$

In this case $\Delta f \rightarrow \infty$ (from Equation 36, 39) and the application of the proposed criterion (Equation 36, 39) has less importance. But the proposed idea plays an important role when the resistivities are of same range i.e.

$$
\rho_{m}^{a} \simeq \rho_{n}^{a}
$$

If situation is like this then we have

$$
\frac{\rho_{n}^{a}}{\rho_{m}^{a}} \rightarrow 1 \quad \text { or } \quad \frac{\rho_{m}^{a}}{\rho_{n}^{a}} \rightarrow 1
$$

Then in this case (from Equation 36, 39).

Minimum evaluation frequency $\left(f_{n}\right)$ used is $1 \mathrm{~Hz}$

\section{Set A}

(frequency separation,

$\left.\Delta f=0.3 f_{n}\right)$

$1,1.3,1.69,2.19,2.86,3.71$,

$4.83,6.28,8.16$,

$10.61,13.79,17.93,23.31$,

$30.30,39.39$,

$51.21,66.57,86.54,112.50$,

$146.25,190.13$

$247.17,321.32,417.72,534.04$

$705.95,917.74$

Table 1: Details of evaluation frequencies used to test the proposed frequency separation condition

$$
\Delta f \rightarrow(\sqrt{2}-1) f_{n}=0.414 f_{n}
$$

So, in real situation when the subsurface is totally unknown the proposed criterion reduces to

$$
\Delta f \leq 0.414 f_{n} \begin{cases}\text { for } & \rho_{m}^{a}>\rho_{n}^{a} \\ \text { for } & \rho_{n}^{a}>\rho_{m}^{a}\end{cases}
$$

Now, applying this frequency separation, we will get the condition for minimum resolvable thickness $(t)$ based on difference of skin depth (Equation 2) as

$$
t>\Delta d
$$

But for layered earth skin depth difference will be

$$
\Delta d=500\left\{\sqrt{\frac{\rho_{n}^{a}}{f_{n}}}-\sqrt{\frac{\rho_{m}^{a}}{f_{m}}}\right\}
$$

So, condition for minimum resolvable thickness will be

$$
t>500\left\{\sqrt{\frac{\rho_{n}^{a}}{f_{n}}}-\sqrt{\frac{\rho_{m}^{a}}{f_{m}}}\right\}
$$

Replacing the values of $\rho_{n}^{a}, \rho_{m}^{a}$ from Equation (22) and $f_{n}, f_{m}$ from Equation (30) we have

$$
t>500\left\{\sqrt{\frac{\left(\frac{\rho^{\prime}}{b_{2}}\right)}{f_{n}}}-\sqrt{\frac{b_{1} \rho^{\prime}}{k^{2}\left(b_{1} b_{2}\right) f_{n}}}\right\}
$$

$$
=500 \sqrt{\frac{\left(\frac{\rho^{\prime}}{b_{2}}\right)}{f_{n}}}\left(1-\frac{1}{k}\right)=\left(\frac{k-1}{k}\right) d_{n}^{a}
$$


Putting the value of ' $k$ ' in Equation (49) we have

$$
t>0.16 d_{n}^{a}
$$

Similary,

$$
\begin{aligned}
& t>500\left\{\sqrt{\left.\frac{\left(\frac{\rho^{\prime}}{b_{2}}\right)}{\left[\frac{f_{m}}{\left(k^{2} b_{1} b_{2}\right)}\right]}-\sqrt{\frac{b_{1} \rho^{\prime}}{f_{m}}}\right\}}\right. \\
& =500 \sqrt{\frac{\rho^{\prime} b_{1}}{f_{m}}}(k-1)=(k-1) d_{m}^{a}
\end{aligned}
$$

Putting the value of ' $k$ ' in Equation (52) we have

$$
t>0.18 d_{m}^{a}
$$

Combining Equations (50) and (53) we have

$$
t>0.169 \sqrt{d_{n}^{a}} \sqrt{d_{m}^{a}}
$$

So, this will be the minimum resolvable thickness criterion with the proposed frequency separation.

\section{Conclusion:}

The prime objective of the study was to enhance the resolution of the subsurface structure and to find the maximum evaluation frequency separation for this resolution enhancement through Magnetotelluric approach. Our study suggests that to enhance the resolution we should take the evaluation frequency separation according to the ratio of corresponding apparent resistivity and the higher frequency should be 1.414 times of the lowest frequency to be used for a particular decade which is multiplied by the resistivity ratio and the minimum resolvable thickness for this frequency separation should be greater than 0.169 times of product of square roots of skin depths. For real field situation, where the resistivities are unknown, the proposed frequency separation criterion reduces to a convenient form where the frequency separation is 0.414 times of the lowest frequency to be taken for a particular decade. During the study equivalent depth for a particular frequency is taken the corresponding skin depth. So, there are opportunities for prospective

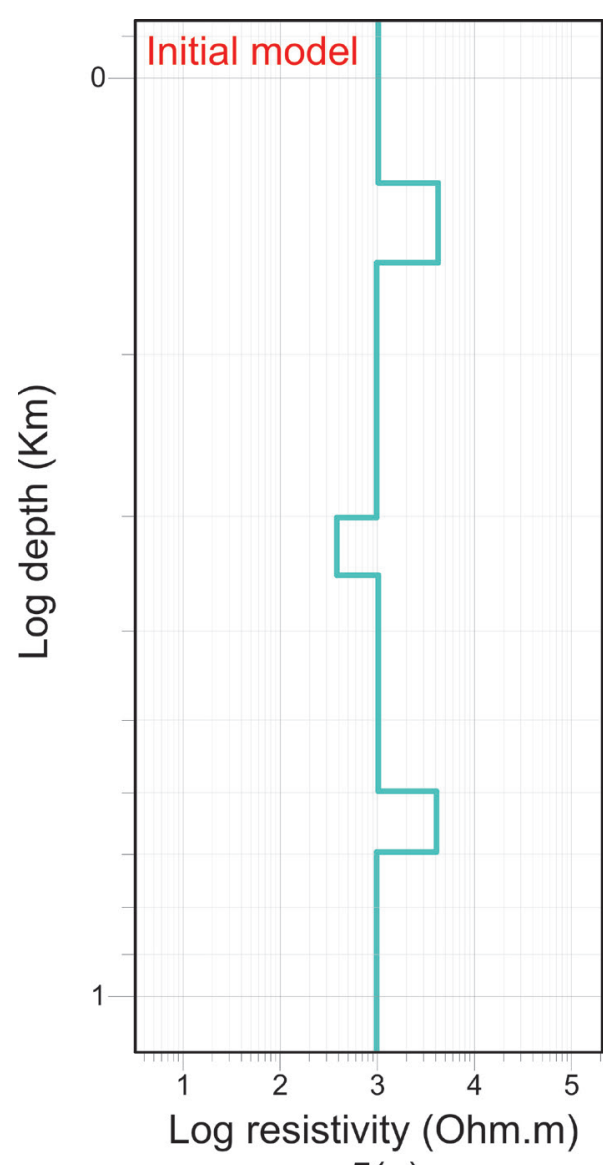

$5(a)$

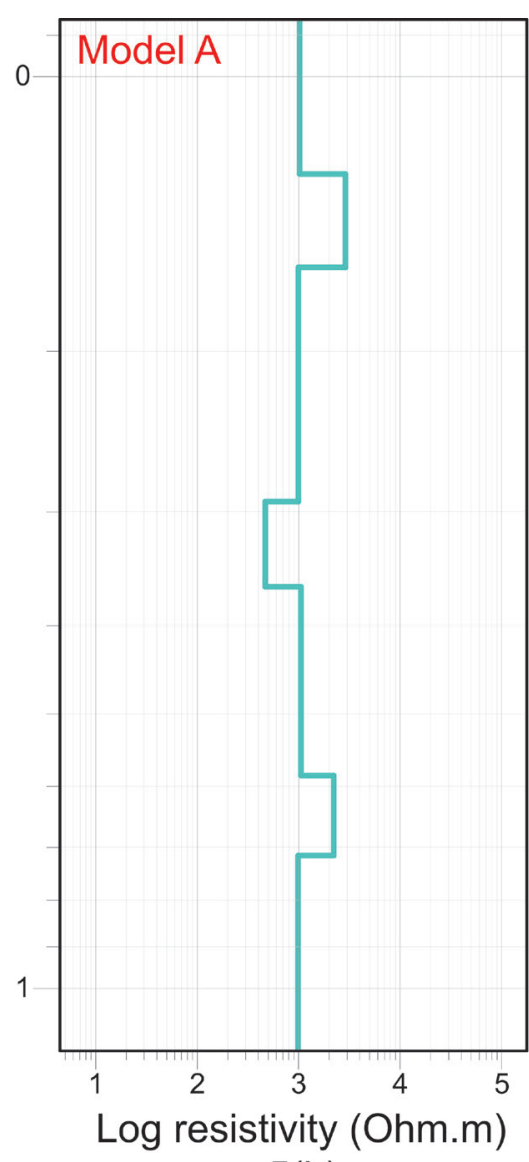

5 (b)

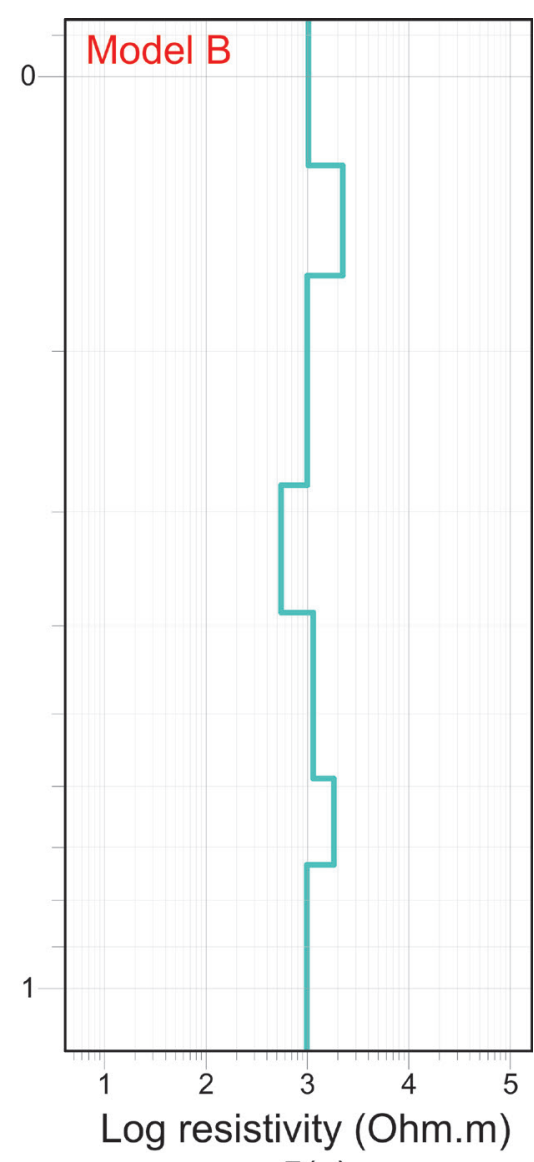

5(c)

Figure 5. Comparisons between the final inverted models (model A and model B) with the initial model to test the effect of evaluation frequency separation on resolution of 1D earth. 5(a) Initial synthetic model to test the evaluation frequency separation condition. 5(b) Final inverted model obtained with evaluation frequency separation taken according to the proposed criterion $\left(\Delta f=0.3 f_{n}\right)$. $5(\mathrm{c})$ Final inverted model obtained with evaluation frequency separation which does not agree the proposed frequency $\operatorname{criterion}\left(\Delta f=0.6 f_{n}\right)$. 
studies of the proposed resolution criteria on the basis of another equivalent depths obtained from other depth transformations such as Bostick equivalent depth.

Acknowledgements. Present study is supported through SHORE project PSC0205 (BPKP) to CSIR-NGRI, Hyderabad. Thanks to the director, CSIR-NGRI for his support and encouragement during the study. Thanks to the anonymous reviewer for his constructive suggestions.

\section{References}

Bedrosian, P.A.(2007). MT+, Integrating Magnetotellurics to Determine Earth Structure, Physical State and Processes, Surveys in Geophysics, 28, 121-167.

Cagniard, L. (1953). Basic theory of the magnetotelluric method of geophysical prospecting, Geophysics, 18, 605-635.

Constable S. C., R. L. Parker and C. G. Constable (1987). Occam's inversion: A practical algorithm for generating smooth models from electromagnetic sounding data, Geophysics, 52, 289-300.

Jones, A. G. (1987). MT and reflection: an essential combination, Geophysical Journal of the Royal Astronomical Society, 89,7-18.

Marquard, D. (1963). An algorithm for least squares estimation of nonlinear parameters, Society for Industrial and Applied Mathematics Journal on Applied Mathematics

Orange, A. S. (1989). Magnetotelluric Exploration for Hydrocarbons, Proceedings of the Institute of Electrical and Electronics Engineers, 77, 267-317.

Peacock, J., S. Thiel, P. Reid, M. Messellier and G. Heinson (2012). Monitoring Enhanced Geothermal Fluids With Magnetotellurics, Test Case: Paralana, South Australia, Proceedings, Thirty-Seventh Workshop on Geothermal Reservoir Engineering Stanford University, Stanford, California.

Simpson, F., andK. Bahr(2005). Practicalmagnetotellurics, Cambridge University Press.

Spies, B. R. (1989). Depth of investigation in electromagnetic sounding methods, Geophysics, 54, 872-88.

Thiel, S., J. Peacock, G. Heinson, P. Reid and M. Messeiller (2010). First results of monitoring fluid injection in EGS Reservoirs Using Magnetotellurics, Australian Geothermal Conference, 259-262.

\section{Appendix A}

\section{Effect of station spacing on horizontal resolution:}

To study the horizontal resolution, we consider the same frequencies $f_{m}$ and $f_{n}$, for a homogeneous medium then the maximum horizontal length that can be imaged below a depth $d_{m}$ will be BC (Figure 1b) on one side of the sounding point. From the Figure (1b) we have

$$
B C=x=\sqrt{\left(A C^{2}-A B^{2}\right)}=\sqrt{\left[\left(d_{m}+\Delta d\right)^{2}-d_{m}^{2}\right]}=\sqrt{d_{n}^{2}-d_{m}^{2}}
$$

For the same homogeneous medium, for two nearby stations A and B (Figure A1) lateral resolvable lengths for both the stations are same i.e.

$$
x_{A}=x_{B}=\sqrt{d_{n}^{2}-d_{m}^{2}}
$$
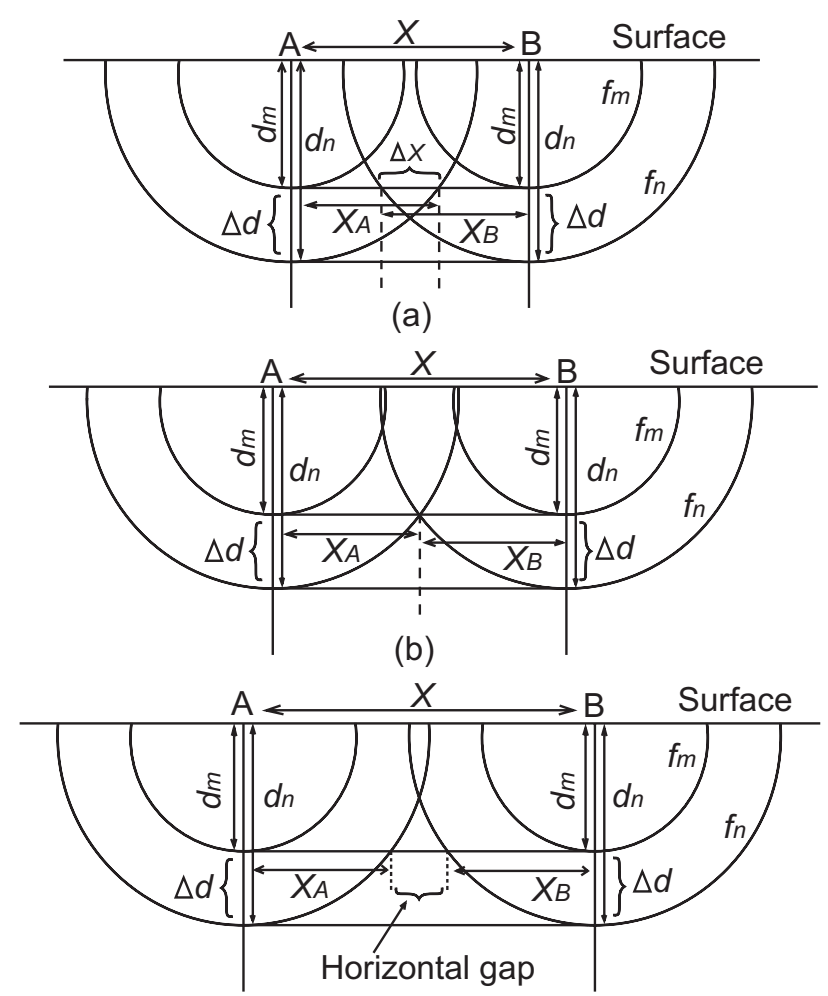

(c)

Figure A1. Overlapping geometry of diffusive hemispheres from two nearby stations $A$ and $B$ below a depth $d_{m}$ (skin depth for frequency $\left.f_{m}\right)$ in homogeneous medium. $f_{n}, f_{m}$ are the evaluation frequencies and $d_{n}, d_{m}$ are the corresponding skin depths. $x$ is the station spacing and $x_{A}, x_{B}$ are the minimum horizontal resolvable length on one side (for homogeneous medium $x_{A}=x_{B}$ ). A1(a) Hemispheres corresponds to frequency $f_{n}$ from two nearby stations $\mathrm{A}$ and B overlap each other below the depth $d_{m}$ with an overlapping amount $\Delta x$ at depth $d_{m}$ A1(b) Hemispheres corresponds to frequency $f_{n}$ just meet each other at the depth $d_{m}$ provides the critical condition. A1(c) Hemispheres corresponds to frequency $f_{n}$ from two nearby sites do not overlap each other below a depth $d_{m}$ producing a horizontal gap at that depth.

In this two stations case the horizontal resolution is related to the overlapping length of hemispheres $(\mathrm{Fi}$ gure A1) at subsurface which is directly related to the station spacing. Here we have three conditions

\section{Condition (a):}

When the intersecting point of the two hemispheres is below the depth $d_{m}$ then some amount of overlapping of the hemispheres will be there below a 
depth $d_{m}$ (Figure A1a).

Then with the combination of two sounding points, the common measurable horizontal length between two stations will be

$$
\Delta x=x_{A}-\left(x-x_{B}\right) \text { or } x_{B}-\left(x-x_{A}\right)
$$

and we have a condition for this situation as

$$
\left(x_{A}+x_{B}\right)-x>0
$$

where $x$ is the station spacing and $x_{A}$ and $x_{B}$ are the maximum horizontal length that can be imaged on one side of the sounding stations at $\mathrm{A}$ and $\mathrm{B}$.

Condition (b):

When the intersection point of two hemispheres just meet (critical point) at that depth $d_{m}$ (Figure A1b) then with combination of two sounding points, the common measurable horizontal length between two stations will be

$$
\Delta x=\left(x_{A}+x_{B}\right)-x=0
$$

Condition (c):

When the hemispheres intersect above a depth $d_{m}$ (Figure A1c) then we will get a horizontal gap below $d_{m}$ which cannot be resolve with that frequencies and station spacing below $d_{m}$ and that gap will be

$$
x-\left(x_{A}+x_{B}\right)
$$

and we have condition for this situation as

$$
\Delta x<0
$$

So, while dealing with the horizontal resolution below certain depth we should take the station spacing for which $\Delta x>0$.

Now to find the optimal station spacing, let us consider $l$ is the length of an object to be resolved below a depth $d_{m}$. Now, if we place the stations at the ends of the object then $l=x$ and in this case condition (b) will provide the minimum overlapping condition i.e.

$$
x_{A}+x_{B}=x=l
$$

and for homogeneous medium

$$
\begin{gathered}
x_{A}=x_{B} \\
\text { So } \quad x_{A}=\frac{l}{2} \text { and } x_{B}=\frac{l}{2}
\end{gathered}
$$

But for better resolution

$$
\begin{gathered}
\Delta x>0 \\
\left(x_{A}+x_{B}\right)-x>0 \\
x<l
\end{gathered}
$$

So, $x$ should be less than $l$.

Now let us consider, we have to resolve a length $L$ with a variable station spacing $x$ such that

$$
L=n x
$$

where $\mathrm{n}$ is a positive rational number

$$
x=\frac{L}{n}
$$

Now from Equation (a8) we have

So,

$$
x_{A}+x_{B}=x=\frac{L}{n}
$$

$$
x_{A}=\frac{L}{2 n} \text { and } x_{B}=\frac{L}{2 n}
$$

But according to the overlapping condition (condition a) we should maintain

$$
\begin{gathered}
\Delta x>0 \\
\left(x_{A}+x_{B}\right)-x>0 \\
\left(\frac{L}{2 n}+\frac{L}{2 n}\right)>x \\
x<\frac{L}{n}
\end{gathered}
$$$$
\text { So, } \quad\left(\frac{L}{2 n}+\frac{L}{2 n}\right)>x
$$

Equation (a21) will satisfy Equation (a18) (i.e. condition a) only if $n$ has any positive rational number greater than 1 . In that case $x$ will be less than $L$. So, the station spacing should always be smaller than the length of the body.

${ }^{\star}$ Corresponding author: Ujjal K. Borah Magnetotellurics and Deep Resistivity Sounding Division CSIR-National Geophysical Research insitute, Hyderabad, India; email: ujjal.borah6@gmail.com

(C) 2017 by the Istituto Nazionale di Geofisica e Vulcanologia. All rights reserved 